\title{
004 EPILEPSY PREVALENCE, INCIDENCE AND SOCIOECONOMIC DEPRIVATION
}

Owen Pickrell, ${ }^{1,2,4}$ Arron Lacey, ${ }^{1,3}$ Owen Bodger, ${ }^{4}$ Joanne Demmler, ${ }^{3}$ Rhys Thomas, ${ }^{1,5}$ Ronan Lyons, ${ }^{3}$ Phil Smith, ${ }^{1,6}$ Mark Rees, ${ }^{1,2,4}$ Mike Kerr ${ }^{1,5}$. ${ }^{1}$ Wales Epilepsy Research Network; ${ }^{2}$ Neurology and Molecular Neuroscience Research Group, College of Medicine, Swansea University; ${ }^{3}$ Farr Institute, College of Medicine, Swansea University; ${ }^{4}$ Institute of Life Sciences, College of Medicine, Swansea University; ${ }^{5}$ Insititute of Psychiatric Medicine \& Clinical Neurosciences, Cardiff University; ${ }^{6}$ Department of Neurology, University Hospital of Wales, Cardiff

\subsection{6/jnnp-2014-309236.4}

Aims To investigate the relationship between socioeconomic deprivation, epilepsy prevalence and incidence.

Method: We reviewed electronic primary health care records in Wales from 2004 to 2010 identifying prevalent and incident cases of epilepsy and recording linked deprivation scores. Logistic and Poisson regression models were used to calculate odds ratios and incidence rate ratios. The change in deprivation was measured ten years after the initial diagnosis of epilepsy for a cohort of people.

Results 8.1 million patient years of records were reviewed. Epilepsy prevalence and incidence were significantly associated with deprivation. Prevalence ranged from $1.12 \%(1.07 \%-$ $1.19 \%)$ in the most deprived decile to $0.49 \%(0.45 \%-0.53 \%)$ in the least deprived decile (adjusted odds ratio $0.92 \mathrm{p}<0.001$ ). Incidence ranged from 40/100000/year in the most deprived decile to 19/100000/year in the least deprived decile (adjusted incidence rate ratio $0.94 \mathrm{p}<0.001)$. There was no significant change in deprivation index decile nine years after a new diagnosis of epilepsy (mean difference $=-0.04, p=0.999$ ). 


\section{ABN Abstracts}

Conclusion Epilepsy prevalence is strongly associated with socioeconomic deprivation. Post-diagnosis 'social drift' is a less likely explanation for this relationship given that incidence is also associated with deprivation and deprivation score does not change ten years after diagnosis. 